\title{
Optical performance of hybrid porous silicon-porous alumina multilayers
}

L. G. Cencha, C. Antonio Hernández, L. Forzani, R. Urteaga, and R. R. Koropecki

Citation: Journal of Applied Physics 123, 183101 (2018); doi: 10.1063/1.5027073

View online: https://doi.org/10.1063/1.5027073

View Table of Contents: http://aip.scitation.org/toc/jap/123/18

Published by the American Institute of Physics

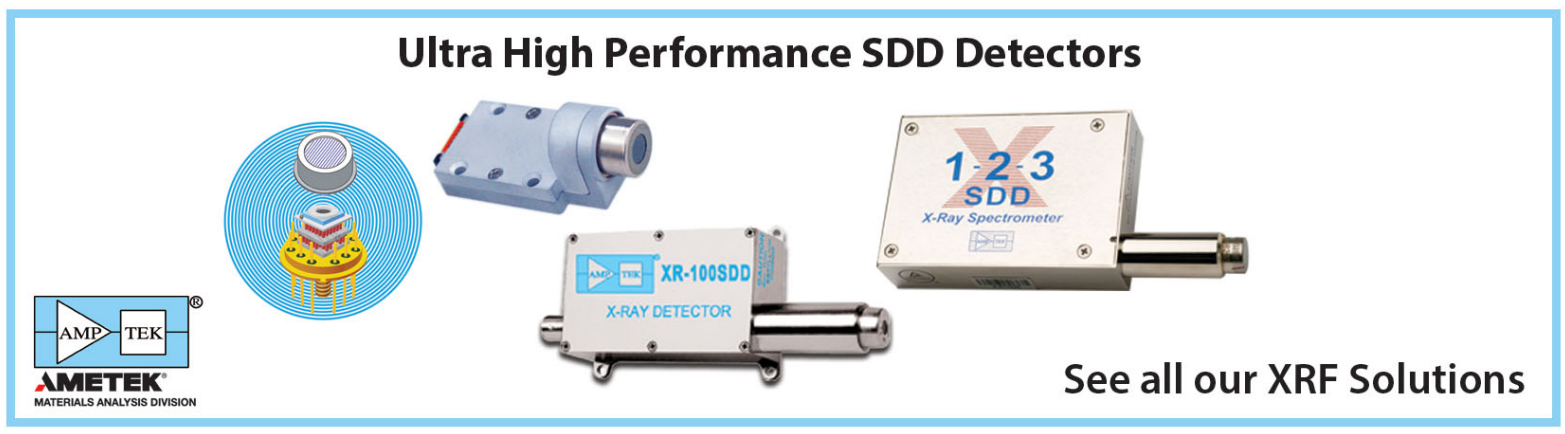




\title{
Optical performance of hybrid porous silicon-porous alumina multilayers
}

\author{
L. G. Cencha, ${ }^{1,2, a), b)}$ C. Antonio Hernández, ${ }^{3}$ L. Forzani, ${ }^{1}$ R. Urteaga,,${ }^{1,2}$ \\ and R. R. Koropecki ${ }^{1,4}$ \\ ${ }_{1}^{1}$ Instituto de Física del Litoral, CONICET-UNL, Güemes 3450, Santa Fe, Argentina \\ ${ }^{2}$ Facultad de Ingeniería y Ciencias Hídricas-UNL, Ciudad Universitaria Ruta Nacional No. 168-Km 472,4, \\ 3000 Santa Fe, Argentina \\ ${ }^{3}$ Centro de Investigación en Dispositivos Semiconductores, Universidad Autónoma de Puebla CIDS-ICUAP, \\ 14 sur and Av. San Claudio, San Manuel, 72570 Puebla, Mexico \\ ${ }^{4}$ Facultad de Ingeniería Química, UNL, Santiago del Estero 2829, 3000 Santa Fe, Argentina
}

(Received 27 February 2018; accepted 25 April 2018; published online 10 May 2018)

\begin{abstract}
In this work, we study the optical response of structures involving porous silicon and porous alumina in a multi-layered hybrid structure. We performed a rational design of the optimal sequence necessary to produce a high transmission and selective filter, with potential applications in chemical and biosensors. The combination of these porous materials can be used to exploit its distinguishing features, i.e., high transparency of alumina and high refractive index of porous silicon. We assembled hybrid microcavities with a central porous alumina layer between two porous silicon Bragg reflectors. In this way, we constructed a Fabry-Perot resonator with high reflectivity and low absorption that improves the quality of the filter compared to a microcavity built only with porous silicon or porous alumina. We explored a simpler design in which one of the Bragg reflectors is replaced by the aluminium that remains bound to the alumina after its fabrication. We theoretically explored the potential of the proposal and its limitations when considering the roughness of the layers. We found that the quality of a microcavity made entirely with porous silicon shows a limit in the visible range due to light absorption. This limitation is overcome in the hybrid scheme, with the roughness of the layers determining the ultimate quality. Q-factors of 220 are experimentally obtained for microcavities supported on aluminium, while Q-factors around 600 are reached for microcavities with double Bragg reflectors, centred at $560 \mathrm{~nm}$. This represents a four-fold increase with respect to the optimal porous silicon microcavity at this wavelength. Published by AIP Publishing. https://doi.org/10.1063/1.5027073
\end{abstract}

\section{INTRODUCTION}

The discovery made by Canham in 1990 about the efficient and tunable luminescence of porous silicon (PS) has attracted great interest and an enormous amount of work in relation to silicon photonics. ${ }^{1}$ This also triggered a number of other studies about the optoelectronic properties of PSi. In particular, in 1994, two pioneering works demonstrated the possibility of making porous silicon multilayers with a specific optical response. ${ }^{2,3}$ The porous nature of the material, connecting each layer of the multilayers with the environment, and the large specific surface area make these structures adequate platforms for chemical sensing and biosensing and tunable filters. These characteristics are extended to anodic aluminium oxide (AAO) membranes. The technological potential of such porous optical materials has been demonstrated in various fields, such as detection and recognition of targeted biological or chemical species, photovoltaics, or radiation shielding. ${ }^{4,5}$ At present, there is great interest in the study and development of label free sensors based on the photonic properties of multilayers made of porous materials such as PS or porous AAO. ${ }^{6-10}$ Some few approaches introduce the use of hybrid structures of porous silicon-polymer to extend the chemical resistance of the devices or the use of a

\footnotetext{
a) Author to whom correspondence should be addressed: luisa.cencha@ santafe-conicet.gov.ar. Tel.: +543424559174.

${ }^{b}$ http://www.ifis.santafe-conicet.gov.ar/integrantes.html
}

polymer optical waveguide to build a two-cascade micro-resonator device. ${ }^{11,12}$ Typically, multilayer structures with optical reflection or transmission spectra having sharp features are used as platforms to construct the sensors. In particular, distributed Bragg reflectors (DBRs), ${ }^{13}$ rugate filters, ${ }^{14}$ or optical microcavities ${ }^{15}$ with sharp resonances in the stopping band are used. Sharp resonances can be obtained by placing a large optical thickness layer between two highly reflective layers (Fabry-Perot resonator). The sharpness of the resonances in the photonic gap is determined by the Q-factor of the structure, defined as $\mathrm{Q}=\frac{\lambda}{\Delta \lambda}$. There are two main limiting features to get a large Q-factor: dispersive effects due to absorption $^{16,17}$ and roughness of the surfaces which produces scattering effects. ${ }^{18,19}$ Optical microcavities made of PS with resonance modes in infrared regions of the spectrum have shown excellent performance due to the low extinction coefficient of porous silicon, implying that the Q-factor is mainly limited by scattering processes. In fact, the high refractive index (RI) modulation that can be obtained with PS makes it an ideal structure for generating strong optical confinement. Sub-nanometer resonances have been obtained for optical microcavities centred on $\lambda_{0}=1.5 \mu \mathrm{m}$ with a cavity of $\lambda_{0}$ optical thickness. ${ }^{16}$ The reported Q-factor in that case was as large as $3400 .{ }^{16}$ Moreover, the low absorption of porous silicon for large wavelengths allows the use of large coupled cavities with low absorption, which can result in multiple resonances with very large Q-factors. However, it is sometimes 
required to tune the sensor in the visible range when, for example, solvents having absorption bands in the infrared range are used or when a naked eye detection is desired. Unfortunately, in the range of visible wavelengths, the large absorption coefficient of porous silicon limits the Q-factor of the microcavities. In contrast, for AAO, the absorption coefficient is very low in a wide range of wavelengths from ultraviolet to infrared. ${ }^{20}$ On the other hand, the refractive index range that can be obtained in AAO is reduced, which makes the obtainment of strong optical confinement difficult. Moreover, the experimental process of tailoring the optical response of porous AAO multilayers is more complicated than in porous silicon routines. In particular, to obtain a DBR using AAO, a selective chemical dissolution of layers must be done after the fabrication of the multilayer. ${ }^{21}$ For this reason, the reported Q-factors for optical multilayers entirely made of AAO are relatively low, reaching values up to $70 .^{22}$

In this work, we study the possibility of combining in a single photonic device the best characteristics of both materials, taking advantage of the low absorption coefficient of porous AAO, and the possibility of obtaining in a simple way a high contrast PS DBR. We propose hybrid structures, in a Fabry-Perot configuration, made of a central AAO cavity and PS DBRs at both sides. In this way, large cavities can be obtained with low absorption, providing a high Q-factor with relatively high transmittance. Moreover, since they are constructed separately, the AAO cavity may be functionalized in a different way than the PSDBRs, allowing a major versatility of the response in the presence of a specific analyte.

In order to optimize the performance of the multilayer, the role of surface roughness in AAO must be taken into account. This is relevant since we found that the optical thickness at each point of a porous AAO membrane slightly depends on the crystal orientation of the underlying aluminium substrate, i.e., the AAO obtained from each crystal of the aluminium substrate has a specific optical thickness. The heterogeneity in the optical thickness introduces a large effective roughness. Therefore, effective surface roughness is increased when areas that span multiple crystals are considered, leading to high scattering effects. This effect modifies the interference behaviour of the device, broadening the spectral bands and decreasing the optical Q-factor of the microcavities. However, the surface roughness of each individual crystal is still small. Therefore, to minimize the problem, we proposed a pretreatment on the aluminium substrate to enlarge the grain size before anodization and use a reduced spot size for the spectroscopic measurements in order to sense a single domain.

In this work, we demonstrate by simulations and experiments that hybrid microcavities can reach larger Q-factors than those obtained with microcavities entirely made of PS in the visible wavelength region.

\section{MATERIALS AND METHODS}

The AAO membranes were fabricated by a two-step anodization process of high purity aluminium foils (99.999\%), $0.02 \mathrm{in}$. of thickness from Laurand associates. ${ }^{23}$ Before the anodizing process, aluminium was pretreated to increase the grain size by thermal annealing and plastic deformation. A first annealing at $400{ }^{\circ} \mathrm{C}$ during $3 \mathrm{~h}$ was followed by mechanical pressing at $7.5 \mathrm{ton} / \mathrm{cm}^{2}$ at room temperature. Finally, a second thermal annealing identical to the first was repeated. Recrystallization and grain growth are the main phenomena occurring during annealing. Within each growing grain, the atoms are lined up in a specific pattern that depends upon the crystal structure of the metal. The cold deformation applied to aluminium provides nuclei and sufficient stored energy to drive the grain growth in the beginning of recrystallization. So, if it is subsequently heated to a sufficiently high temperature after deformation, new dislocation-free grains will be formed within the deformed material, which leads to a progressive increase in the grain size. ${ }^{24}$

The treated aluminium substrates were then electropolished for 8 min under a constant voltage of $20 \mathrm{~V}$, in $1: 4$ mixture of perchloric acid $\left(\mathrm{HClO}_{4}\right)$ and ethanol $(\mathrm{EtOH})$ to obtain a flat surface. Then, the first electrochemical anodization was performed at $7{ }^{\circ} \mathrm{C}$ by applying $40 \mathrm{~V}$ to the electropolished $\mathrm{Al}$ films in a solution of $0.3 \mathrm{M}$ oxalic acid $\left(\mathrm{H}_{2} \mathrm{C}_{2} \mathrm{O}_{4}\right)$ for $20 \mathrm{~h}$. Next, the aluminium oxide films with disordered pores were dissolved by wet chemical etching using a mixture of $0.4 \mathrm{M}$ phosphoric acid $\left(\mathrm{H}_{3} \mathrm{PO}_{4}\right)$ and $0.2 \mathrm{M}$ chromic acid $\left(\mathrm{H}_{2} \mathrm{CrO}_{4}\right)$. Then, the second anodization step was performed under the same anodization conditions as the first one and was carried out until the nanostructured AAO layers reached the desired thicknesses. Both the electropolishing and the anodization steps were carried out under stirring. Thereby, hexagonally distributed pore arrays with a $40 \mathrm{~nm}$ pore diameter and a $120 \mathrm{~nm}$ pore distance were obtained.

The porous silicon layers were fabricated by electrochemical anodization of p-type boron doped c-Si wafers (resistivity 1-4 $\mathrm{m} \Omega \cdot \mathrm{cm}$, orientation [100]) in an $\operatorname{HF}(50 \%)$ / EtOH solution with proportions 1:2 (v:v). The electrochemical cell is a Teflon ${ }^{\circledR}$ beaker where the Si wafer acts as an anode and the cathode is formed by a platinum wire. Using this cell, PS is formed on the wafer surface exposed to HF. Two kinds of layers were prepared at constant current densities of 5 and $70 \mathrm{~mA} / \mathrm{cm}^{2}$ in the galvanostatic mode. The porosities of these films are $65 \%$ and $84 \%$, with pore sizes of about 5 and $40 \mathrm{~nm}$, respectively. After fabrication, an electropolishing pulse with a current density of about $200 \mathrm{~mA} / \mathrm{cm}^{2}$ in an HF (50\%)/EtOH solution with proportions 1:7 (v:v) was applied to remove PS multilayers from the c-Si substrate. The values of single layer porosities and thicknesses were estimated from the fitting of reflectance spectra in the range of $200-1100 \mathrm{~nm}$ using Looyenga mixing rules. ${ }^{25,26}$ These estimated values allow the design of the PS-DBRs, which are experimentally obtained alternating the two different current densities during the anodization process.

Since typical pore sizes in both PS and AAO are smaller than the wavelength of VIS-NIR light, the control of porosity allows the manipulation of their optical properties as effective media. This fact allows the designing and manufacturing of multilayers with specific optical responses by just varying the fabrication parameters (current densities and times) in the anodization processes.

As mentioned earlier, in this work, the optical responses of two kinds of multilayers are presented, one of them composed of a PS-DBR onto a porous AAO membrane supported 
on the aluminium substrate from which it was made [type A, Fig. 1(a)]. The other type of microcavity consists of a sequence of a PS-DBR, a porous AAO central cavity, and a second PS-DBR [type B, Fig. 1(c)]. The difference between both configurations is that in type $\mathrm{A}$, one mirror is the aluminium substrate, while in type $\mathrm{B}$, the mirror is another PS-DBR.

To fabricate the hybrid structures, the first step after the fabrication of the AAO consists of depositing the freestanding PS-DBR over the porous alumina using a gentle traverse flow of isopropyl alcohol to transfer it from the crystalline silicon substrate. When the sample is dried with pure nitrogen, the action of capillary forces on PS-DBR tightly attaches the membrane to the alumina. In this way, the type A microcavity [Fig. 1(a)] is obtained. To obtain a type B microcavity, the remaining aluminium substrate needs to be removed from the bottom side by wet chemical etching in a saturated solution of cupric chloride and hydrochloric acid $\left(\mathrm{CuCl}_{2} / \mathrm{HCl}\right)$. Finally, a second PS-DBR is deposited on the remaining surface of the freestanding AAO sample to obtain the hybrid microcavity [Figs. 1(b) and 1(c)].

The transmittance and reflectance spectra of the hybrid structures were measured using a UV-VIS-NIR (Ocean Optics HR4000) spectrometer with a motorized XY scanning stage which allows point-to-point measurements. The optical thickness of each point of the membranes, i.e., the product between the physical thickness and the effective refractive index of the film, was obtained using reflectometric interference Fourier transform spectroscopy (RIFTS). ${ }^{27}$ The optical thickness was measured with a spatial resolution of about $200 \mu \mathrm{m}$, limited by the light spot size, which is in turn defined by the optical fiber diameter $(300 \mu \mathrm{m})$. The spectral

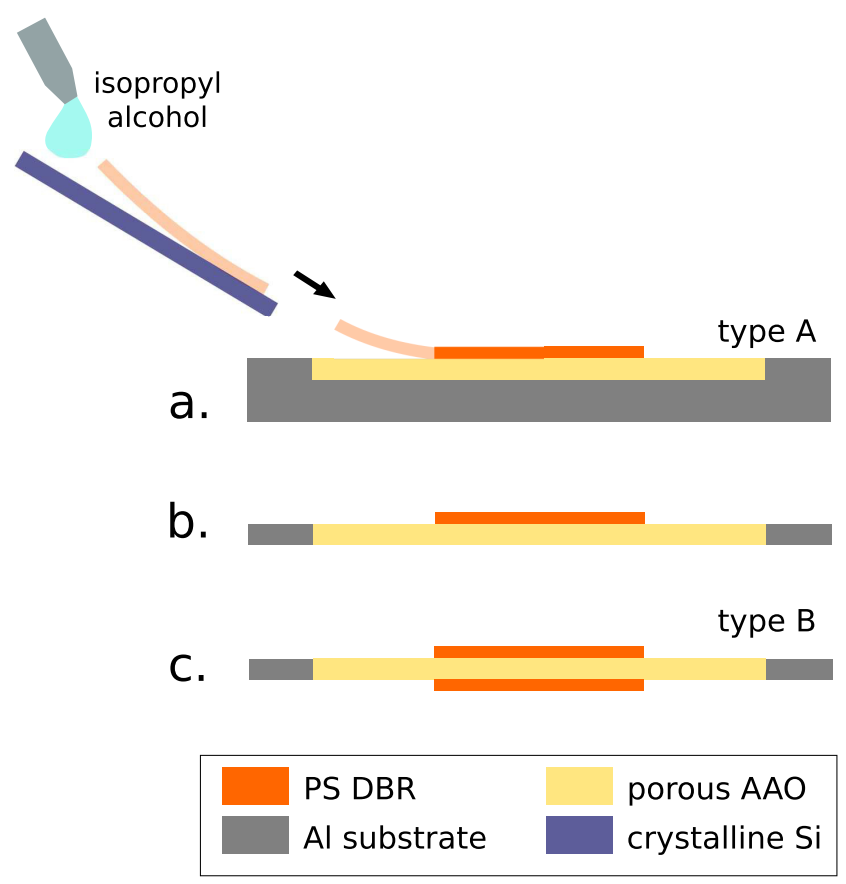

FIG. 1. Scheme for the fabrication of hybrid microcavities. (a) A freestanding PS Bragg reflector is deposited over the surface of AAO using a flow of isopropyl alcohol, then (b) the aluminium substrate is removed by wet chemical etching, and (c) the same procedure as in (b) is repeated to obtain the optical hybrid microcavity. resolution of the system was experimentally determined by measuring the response to a monochromatic source (He-Ne Melles Griot Laser). This spectral response of about $1 \mathrm{~nm}$ full width at half maximum (FWHM) was used to correct the simulated reflectance and transmittance spectra. The spectral resolution of the system limits the maximum Q-factor that can be measured, but it does not represent a limitation in the sensitivity or in the detection limit (DL) if the sensor is constructed using a nondispersive appropriate scheme. ${ }^{28}$ The optical response for hybrid structures was simulated considering the Looyenga effective media model ${ }^{25}$ to calculate the refractive indexes of the layers and using the matrix theory for the light propagation in dielectric thin film multilayer structures. $^{29}$

\section{THEORETICAL ANALYSIS}

In this section, we analyze the performance of the silicon-alumina hybrid microcavities in the context of their use as a resonant refractive index sensor. Such sensors are based on the shift of an optical resonance due to the change in the refractive index (RI) produced in the multilayer. For biomolecule detection applications, for example, the specific capture of biomolecules at the sensor surface results in a local change in RI, producing a sensing signal that enables quantification of the biomolecules in the sample. ${ }^{30-32}$ The performance of these sensors is typically characterized by their sensitivity (S) and their detection limit (DL). ${ }^{17}$ The sensitivity measures the ratio between the resonance position change $(\Delta \lambda)$ and the RI variation $(\Delta n)$. For many resonantbased RI sensors, the sensitivity can be calculated as

$$
S=\frac{\Delta \lambda}{\Delta n}=\eta \frac{\lambda}{n_{e f f}},
$$

where $\eta$ is the fraction of optical intensity in the sample and $\mathrm{n}_{\text {eff }}$ is the effective RI experienced by the resonant mode. ${ }^{17}$ On the other hand, DL represents the smallest sample RI change that can be accurately measured. For biomolecule sensing, for example, the DL is related to the minimum amount of analyte that the RI sensor can accurately measure. Furthermore, the DL can be calculated as $\mathrm{DL}=\mathrm{R} / \mathrm{S}$, where $\mathrm{R}$ is the system resolution, which characterizes the smallest possible spectral change that can be accurately measured.

The value of $\mathrm{R}$ is limited by different factors associated with the particular measurement system. Among these factors are the noise associated with the measurement of peak amplitude and the spectral indetermination due to thermal-induced fluctuations or spectral resolution of the measuring instrument. If the resolution is dominated by the presence of noise in the measured amplitude, the peak position determination presents an uncertainty that limits the value of $\mathrm{R}$. Considering a resolution given by three times the value of the peak position variance $\sigma$, for a typical Lorentzian peak profile, the value of $\mathrm{R}$ can be approximated by

$$
R=3 \sigma \approx \frac{2 \Delta \lambda}{3\left(\mathrm{SNR}^{\frac{1}{4}}\right)},
$$


where $\Delta \lambda$ is the FWHM of the mode amplitude and SNR is the signal-to-noise ratio defined as the quotient between the signal amplitude and the variance of the noise distribution. ${ }^{17}$ Then, by combining these expressions, the following equation is obtained:

$$
D L=\frac{R}{S}=\frac{2 n_{\text {eff }}}{3 \eta\left(\mathrm{SNR}^{\frac{1}{4}}\right) \mathrm{Q}},
$$

where $\mathrm{Q}$ is the $\mathrm{Q}$-factor of the resonant mode, defined as $\mathrm{Q}=\lambda / \Delta \lambda$. Equation (3) implies that the DL of the sensor can be improved by increasing the Q-factor of the resonance mode. Instead, the effect of noise is more subtle. Typically, the noise may have different contributions, some of which may be proportional to peak intensity. However, it is generally true that an increase in peak intensity produces an increase in SNR. Therefore, to obtain a small DL, large peak intensity is desirable. In practical terms, this means that the transmittance of a microcavity must be as high as possible. ${ }^{28,33}$

Moreover, the value of the Q-factor depends on many factors that limit it. The total intrinsic Q-factor of an optical mode is calculated from $1 / \mathrm{Q}_{\text {total }} \approx 1 / \mathrm{Q}_{\text {absorption }}+1 / \mathrm{Q}_{\text {scattering }}$ $+1 / \mathrm{Q}_{\text {radiation, where }} 1 / \mathrm{Q}_{\text {absorption }}$ denotes the losses of photons by material absorption, $1 / \mathrm{Q}_{\text {scattering }}$ represents the scattering losses due to inhomogeneities or surface defects, and $1 / \mathrm{Q}_{\text {radiation }}$ is related to the radiative losses. ${ }^{34}$

Using the transfer-matrix method, the propagation of light through the hybrid microcavity structure can be simulated. ${ }^{29,35}$ Numerical calculations of the transmission spectra have been used to obtain the Q-factor. The Q-factor was simulated as a function of the central cavity width and the number of DBR periods. Type A and type B configurations were simulated considering two cases: the central cavity made of porous AAO (hybrid) and the central cavity made of PS. The simulated curves are shown in Figs. 2(a) (type A microcavity) and 2(b) (type B microcavity), where the blue curves indicate a central cavity made of porous AAO and the red ones indicate a PS central cavity. In this figure, the selected porosities of PS are $65 \%$ and $84 \%$, corresponding to the high and low refractive index layers, respectively.

It can be observed that, when the central cavity is made of porous AAO, the Q-factor increases with the cavity width and with the period number of the DBR in both types, A and $\mathrm{B}$ microcavities. When there is no absorption, the Q-factor of a Fabry-Perot resonator depends only on the side mirrors' reflectance. ${ }^{36,37}$ For this reason, the Q-factor increases with the DBR's period number when the central cavity is made of alumina. The increase in $\mathrm{Q}$ is not observed for the case of a PS central cavity due to the large absorption of silicon. The limit of the Q-factor due to absorption is given by

$$
Q_{\text {absorption }}=\frac{2 \pi n}{\alpha \lambda},
$$

where $\alpha$ is the absorption coefficient and $n$ is the refractive index.

As mentioned, to obtain a small detection limit, it is also desirable to have a high transmittance amplitude besides a high Q-factor. For this reason, the transmittance amplitude was also calculated for a type B microcavity [Fig. 2(c)]. The peak transmittance decreases considerably when the number of DBR periods increases, while the Q-factor increases slightly. The conclusion of the above analysis is that there is a competition between the width of the resonance peak and its transmittance, so that it is convenient to choose a moderate number of periods.

The Q-factor shows saturation in the case of PS microcavities for a high cavity thickness and a high number of Bragg periods and a rapid decrease in the peak transmittance. In the hybrid schemes, the Q-factor is almost proportional to the cavity thicknesses whereas the peak transmittance is almost independent of the cavity thicknesses and decreases steeply with the number of periods in the PS-DBRs.

The quality of a microcavity also depends on the homogeneity of the optical thickness of the central cavity. Different optical thicknesses produce resonances at different wavelengths. These multiple resonances result in a broadening of the spectral features leading to a lower Q-factor. As mentioned earlier, the optical thickness at each point of an AAO porous membrane depends on the crystal structure of the underlying aluminium substrate, as will be shown in Sec. IV. We evaluate the influence of this effect numerically using a Monte Carlo simulation. We used this technique to determine the resultant Q-factor of the resonance mode when the optical thickness is considered to have a normal distribution of relative variance $\sigma_{\text {rug }}$.

If the optical thickness variations are small relative to the total thickness, the transmittance spectrum of the
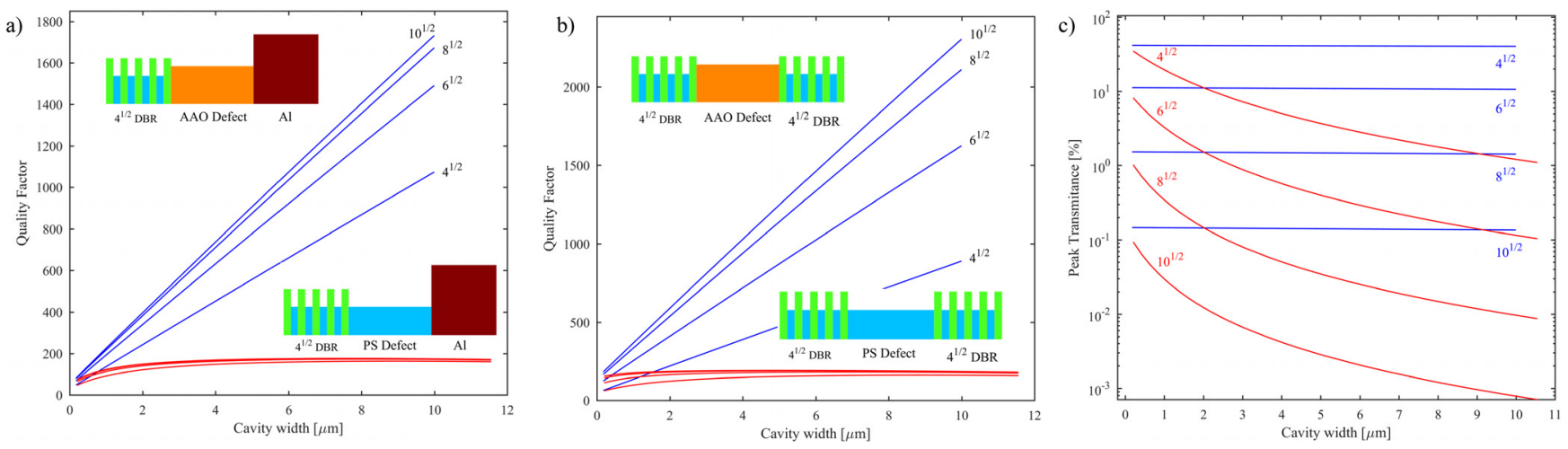

FIG. 2. Comparison of the theoretical Q-factor of hybrid (blue) and PS (red) microcavities with different numbers of Bragg reflector periods (4.5 to 10.5). The Q-factor is shown for type A (a) and type B (b) configurations as a function of the cavity width. The insets show the refractive index profile for the hybrid and porous silicon microcavities. (c) Theoretical peak transmittance of the type B configuration. 


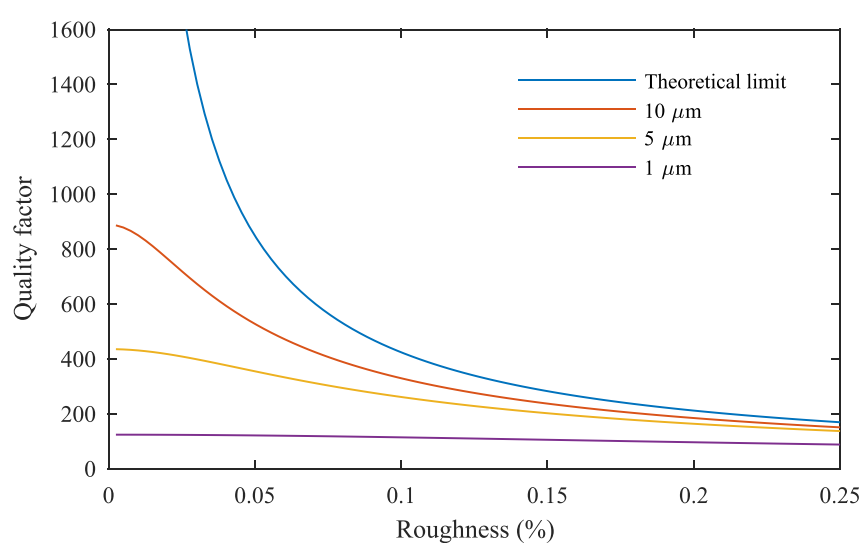

FIG. 3. Q-factor of a hybrid microcavity (type B) constructed with DBRs of 4.5 periods and different thicknesses of $\mathrm{AAO}$ as a function of the roughness of the AAO layer. The roughness value represents the relative standard deviation of the thickness of the AAO layer. The theoretical limit of considering an initial transmission peak of negligible width is also shown.

microcavity will be modified in a very small fraction. According to simple scaling rules, if the change in the optical thickness is small enough, the change in the transmittance spectrum may be approximated by a rigid translation in the same proportion. ${ }^{38}$ This property can be used to approximate the roughness effect as a simple convolution of the spectrum with a peak distribution having a width $\lambda_{0} \frac{\sigma_{\text {rug }}}{L}$, where $\mathrm{L}$ is the cavity thickness. Figure 3 shows the result of calculating the effect of the roughness on the Q-factor for a type B microcavity with DBRs of 4.5 periods for different AAO thicknesses. We used here a roughness with a normal distribution and standard deviation as a percentage of the AAO cavity thickness for each case. Figure 3 also shows the theoretical limit obtained considering an initial transmittance peak broadened only by roughness effects, i.e., with negligible width. This indicates the maximum value of Q-factor that can be obtained by only considering the effect of the roughness.

The obtained results indicate that a relative small roughness of about $0.1 \%$ limits the highest attainable quality factor up to around 400 , irrespective of the cavity thickness.

\section{RESULTS AND DISCUSSION}

In order to improve the optical quality of the porous $\mathrm{AAO}$, the aluminium substrates were pre-treated before the anodization procedure to reduce the effective roughness as described in Sec. II. Figure 4(a) shows a micrograph of an electropolished pure aluminium foil as-received from the supplier. The electropolishing procedure reveals grain boundaries that can be observed as bright lines in dark field microscopy. Typical grain sizes are around $200 \mu \mathrm{m}$, elongated in the rolling direction. The average grain size can be considerably increased applying to $\mathrm{Al}$ substrates the pre-treatment procedure. Figure 4(b) shows an Orientation Image Microscopy (OIM) from reflected electron diffraction (Kikuchi method) of the aluminium foil after applying the pre-treatment procedure. In this image, each colour represents a particular crystal orientation. The well defined crystalline directions are observed in Fig. 4(b), spanning regions as large as $2 \mathrm{~mm}$. Figure 4(c) shows the optical thickness topography of the AAO membrane obtained by anodizing the same aluminium substrate as that of Fig. 4(b). Comparing Figs. 4(b) and 4(c), it can be noticed that each particular grain (labelled with different numbers) in the $\mathrm{Al}$ substrate produces an area with a definite optical thickness in the alumina membrane. It is worth noting that DRX analysis shows that AAO membranes are amorphous, a fact that it is well reported in bibliography. ${ }^{39}$ However, the variation in the optical thickness of the AAO as a result of aluminium crystalline orientation has not been reported before. This
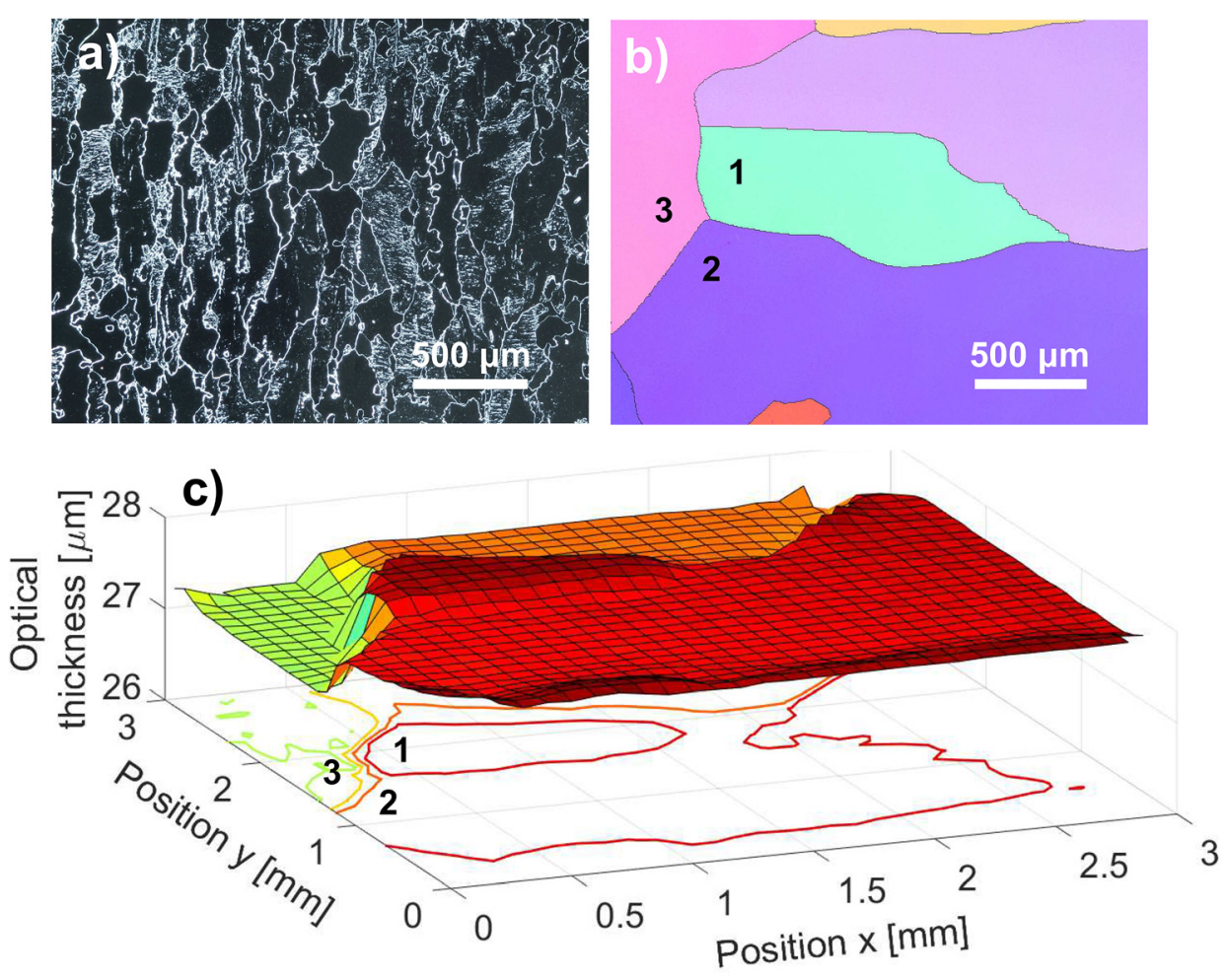

FIG. 4. (a) Dark field micrograph of an electropolished as-received pure aluminium foil. (b) Orientation Image Microscopy from reflected electron diffraction (Kikuchi) of the aluminium foil after the pre-treatment procedure. The substrate was annealed at $400{ }^{\circ} \mathrm{C}$ during $3 \mathrm{~h}$, pressed at $7.5 \mathrm{ton} / \mathrm{cm}^{2}$ and annealed again. (c) Optical thickness of the alumina membrane obtained from the aluminium foil shown in (b). 
a)

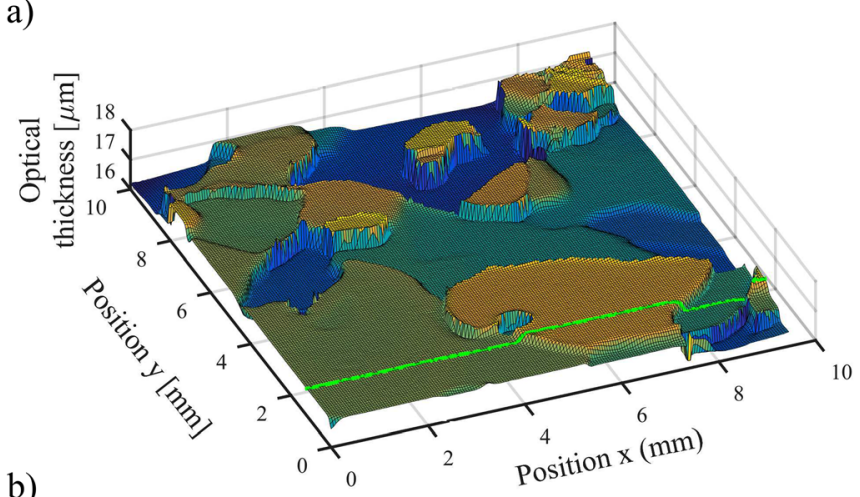

b)

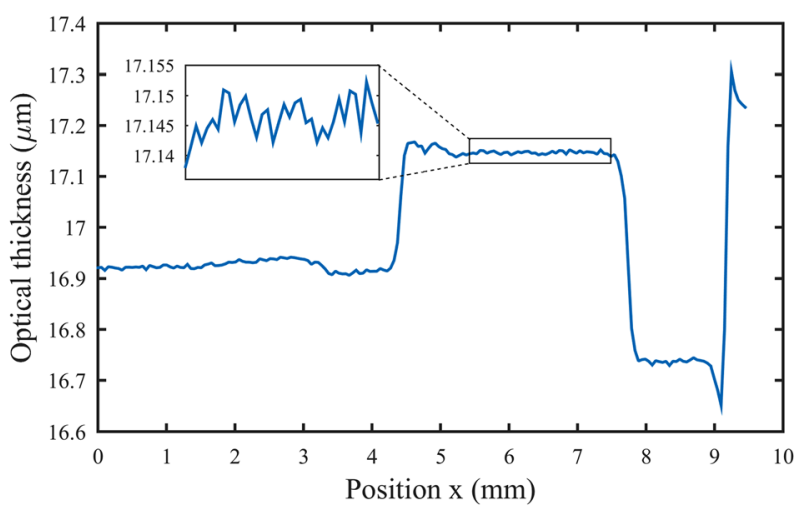

FIG. 5. (a) Topographic representation of the optical thickness of a nanoporous AAO sample in a large area region. (b) The optical thickness profile along the green line shown in Fig. 5(a). Details of the surface roughness over one single crystal are shown in the inset.

slight variation of about $1 \%$ (root mean square) is not relevant in most applications but represents a key limitation to obtain a high quality microcavity (see Fig. 3).

The setup for measurements of reflectance and transmittance of the optical microcavities involves a light beam of about $300 \mu \mathrm{m}$ in diameter. Heterogeneity in the optical thickness of the membrane within the light spot results in a broadening of the resonance peak of the microcavity due to the mix of interference effects coming from regions with different optical thicknesses. As a consequence, the Q-factor decreases as the roughness increases which can be observed in Fig. 3. For this reason, it is desired to achieve the largest grain sizes in the AAO membranes which is possible by a pre-treating of the pure $\mathrm{Al}$ foils.
Figure 5(a) shows a topological view of the optical thickness in an AAO membrane fabricated after the pretreatment of the aluminium foil. The regions of uniform optical thickness have dimensions of about $4 \times 4 \mathrm{~mm}$. Therefore, the light beam can easily probe regions with a uniform optical thickness in a single domain. It can be observed in the profile of Fig. 5(b) that the optical thickness can change steeply up to $3 \%$ between adjacent domains. However, it is worth noting that the rms roughness within a particular grain can be as low as $4 \mathrm{~nm}$ [see inset in Fig. 5(b)]. These measurements were also confirmed by atomic force microscopy in a small region of a single domain. This represents only $0.04 \%$ of the total thickness of the AAO and extends the maximum Q-factor that can be reached up to around 1000 in ideal conditions and around 600 for a $10 \mu \mathrm{m}$ AAO layer (Fig. 3).

Figure 6 shows SEM images of a hybrid type B microcavity consisting of a central layer AAO of $10.7 \mu \mathrm{m}(40 \mathrm{~nm}$ pore diameter and about $10 \%$ porosity) and PS DBR of 6 periods on both sides. The PS DBR is constructed by alternating layers of $65 \%$ and $84 \%$ of porosity, with pore sizes of about 5 and $40 \mathrm{~nm}$, and resulting refractive index values of 1.9 and 1.4 at $560 \mathrm{~nm}$, respectively.

Figure 7 shows experimental results of the reflectance of a hybrid microcavity (type A), constructed with a 4.5 period DBR tuned at $550 \mathrm{~nm}$ and a $10 \mu \mathrm{m}$ AAO layer (see inset). It is important to note that in this type of microcavity, the transmittance measurement is not possible because of the aluminium substrate. The reflectance spectrum was fitted using the AAO thickness and the layer roughness as free parameters. The best fit of the data in Fig. 7 is obtained considering a $0.15 \%$ roughness in the AAO layer. The simulated spectrum has been calculated taking into account the spectral resolution of the spectrometer.

The measured Q-factor value at the central wavelength $(560 \mathrm{~nm})$ is about 220 . In this case, the spectral resolution of the spectrometer does not significantly affect the measured Q-factor value.

Figure 8 shows experimental results of the transmittance of a hybrid microcavity (type B), constructed with two DBRs with 4.5 periods tuned at $560 \mathrm{~nm}$ and a $10.7 \mu \mathrm{m}$ AAO cavity layer. The transmittance spectrum was fitted using the AAO thickness and the layer roughness as free parameters. The best fit of the data in Fig. 8 is obtained considering a a.

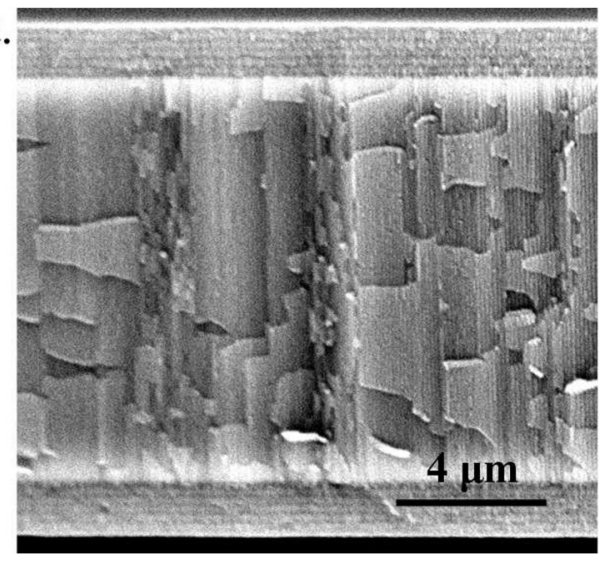

b.

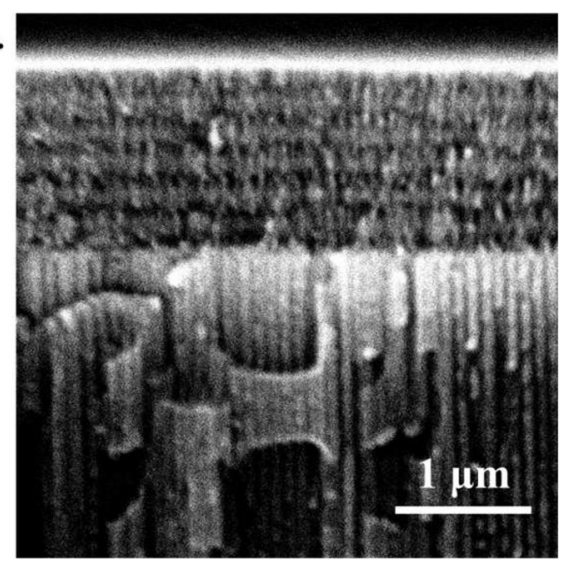

FIG. 6. Cross-sectional SEM micrograph of a hybrid microcavity formed by a central layer of alumina and two porous silicon distributed Bragg reflectors on both sides (a). An enlarged view of the porous silicon reflector where each DBR consists of 6 periods of low and high porosity layers (b). 


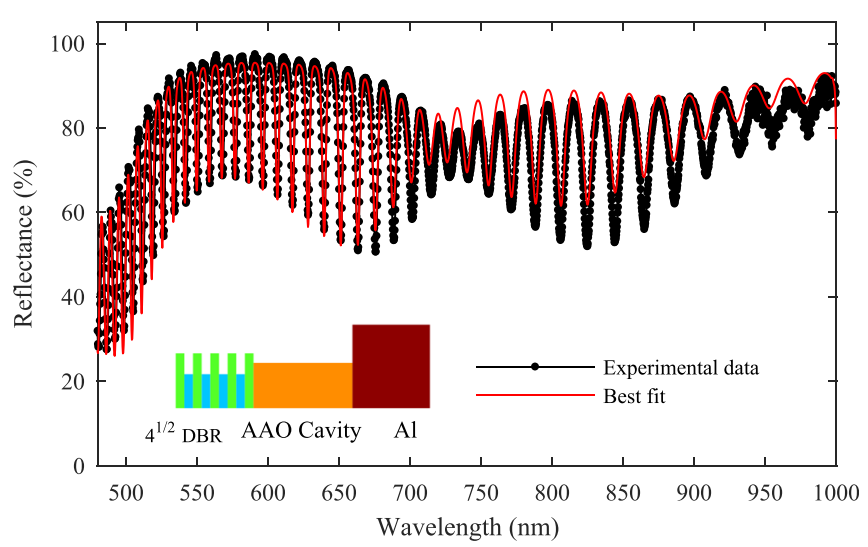

FIG. 7. Measured reflectance data (line and symbols) of a hybrid microcavity (type A) and the best fit taking into account the cavity layer roughness and the spectral resolution of the measuring system (continuous line). The measured Q-factor at the central wavelength $(560 \mathrm{~nm})$ is about 220 . The inset shows a schematic description of the multilayer layout.

$0.04 \%$ roughness in the AAO layer in agreement with the values obtained in Fig. 5.

The measured Q-factor value at the central wavelength $(560 \mathrm{~nm})$ is about 320 with a peak transmission of about $20 \%$. In this case, the spectral resolution of the spectrometer leads to a sizeable contribution to the broadening of the measured resonance peak. After the deconvolution of the measured resonance peak, the resulting Q-factor is about 600, according to the expected value from Fig. 3 and a $0.04 \%$ cavity roughness.

For practical realizations of a sensor, the limited spectral resolution of the detector can be overcome using a second microcavity and measuring the total transmittance intensity of the ensemble. ${ }^{28}$ This second microcavity is not exposed to the analyte and acts as a reference filter. In this way, a shift in the transmittance spectrum of the first microcavity produces a change in the transmitted intensity after the second microcavity. This results in a sensor that requires neither a wavelength-sensitive detector nor a monochromatic source of illumination and is robust to changes in temperature because it only depends on the relative changes in the microcavities. $^{28}$

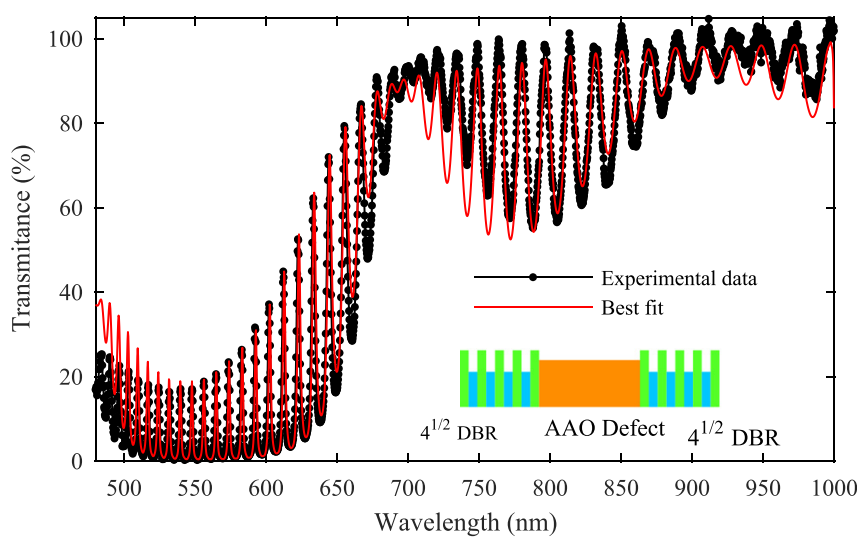

FIG. 8. Experimental transmittance data (line and symbols) of a hybrid microcavity (type B) and the best fit taking into account the cavity layer roughness and the spectral resolution of the measuring system (continuous line). The measured Q-factor at the central wavelength $(560 \mathrm{~nm})$ is about 320. The inset shows a schematic description of the multilayer layout.
It is important to note that whatever the optical thickness of the cavity, a microcavity made entirely of porous silicon, even in the ideal case with zero roughness, cannot reach a Qfactor value greater than 180 at this wavelength, as can be seen in Fig. 2(b).

In order to assess the viability of the hybrid structure as a sensing material, it is important to test the interconnectivity of the porous networks between the different layers. To evaluate this issue, the infiltration of a liquid (isopropyl alcohol) in the hybrid structure was tested by simple capillarity. ${ }^{40}$ The transmittance spectrum of the porous membrane was measured at real time during the liquid infiltration. The spectrum shifts towards longer wavelengths as is expected due to the increase in the effective refractive index in the central cavity. A shift of about $2.8 \%$ in the central peak position is measured when the structure is infiltrated. This shift is in excellent agreement with the expected change of the effective refractive index of porous alumina assuming complete wetting $(2.7 \%)$.

\section{CONCLUSIONS}

In this work, we demonstrate the possibility of constructing hybrid microcavities of porous silicon and porous alumina that take advantage of the individual characteristics of each material. In this way, it has been possible to obtain microcavities with Q-factors up to 600 in the range of wavelengths corresponding to the visible spectrum. Microcavities made with only one of these materials in this range of wavelengths cannot exceed Q-factor values of 170 due mainly to the absorption that silicon presents in the visible range. We have found that the limiting factor in the optical quality of the hybrid microcavities is the roughness of the porous alumina layer forming the cavity. A critical effect is that the optical thickness of the alumina layer depends on the crystalline orientation of the aluminium substrate. Although very small $(\sim 1 \%)$, the deviations from the average optical thickness limit the maximum value of the Q-factor that can be obtained. In this work, we use a thermal annealing and plastic deformation pre-treatment that allows increasing the size of the aluminium crystals prior to the fabrication of the alumina. In this way, we reduce the effective roughness up to $0.04 \%$ in a single crystalline domain. With these roughness values, it would be possible to construct microcavities with $\mathrm{Q}$ values of up to about 1000 in the range of wavelengths corresponding to the visible spectrum. In addition, the construction of hybrid microcavities allows functionalizing in a differentiated way the constituent parts, increasing the versatility of the device to the detection of specific analytes. Finally, the high Q-factor, in combination with a large transmittance at the microcavity resonance, is essential to obtain a sensor with an improved sensitivity and detection limit.

\section{ACKNOWLEDGMENTS}

The authors acknowledge financial support from the CONICET Grant No. PICT 20141683 and from the Universidad Nacional del Litoral, Argentina. C. Antonio Hernandez acknowledges the support received from CONACYT, Mexico. 
${ }^{1}$ L. T. Canham, Appl. Phys. Lett. 57, 1046 (1990).

${ }^{2}$ G. Vincent, Appl. Phys. Lett. 64, 2367 (1994).

${ }^{3}$ M. G. Berger, C. Dieker, M. Thönissen, L. Vescan, H. Lüth, H. Münder, W. Theiss, M. Wernke, and P. Grosse, J. Phys. D: Appl. Phys. 27, 1333 (1994).

${ }^{4}$ M. E. Calvo, S. Colodrero, N. Hidalgo, G. Lozano, C. López-López, O. Sánchez-Sobrado, and H. Míguez, Energy Environ. Sci. 4, 4800 (2011).

${ }^{5}$ E. Osorio, R. Urteaga, L. N. Acquaroli, G. García-Salgado, H. Juaréz, and R. R. Koropecki, Sol. Energy Mater. Sol. Cells 95, 3069 (2011).

${ }^{6}$ F. A. Harraz, M. S. Salem, T. Sakka, and Y. H. Ogata, Electrochim. Acta 53, 3734 (2008)

${ }^{7}$ M. A. Krepker and E. Segal, Anal. Chem. 85, 7353-7360 (2013).

${ }^{8}$ A. Santos, T. Kumeria, and D. Losic, Mater. (Basel). 7, 4297 (2014).

${ }^{9}$ V. Mulloni and L. Pavesi, Appl. Phys. Lett. 76, 2523 (2000).

${ }^{10}$ A. M. Md Jani, D. Losic, and N. H. Voelcker, Prog. Mater. Sci. 58, 636 (2013).

${ }^{11}$ P. Azuelos, P. Girault, N. Lorrain, L. Poffo, I. Hardy, M. Guendouz, and M. Thual, J. Appl. Phys. 121, 144501 (2017).

${ }^{12}$ L. De Stefano, L. Rotiroti, E. De Tommasi, I. Rea, I. Rendina, M. Canciello, G. Maglio, and R. Palumbo, J. Appl. Phys. 106, 023109 (2009).

${ }^{13}$ G. Barillaro, in Handbook of Porous Silicon, edited by L. Canham (Springer, The Netherlands, 2014), p. 845.

${ }^{14}$ M. J. Sweetman and N. H. Voelcker, RSC Adv. 2, 4620 (2012).

${ }^{15} \mathrm{G}$. Shtemberg and E. Segal, in Handbook of Porous Silicon, edited by L. T. Canham (Springer, 2014), pp. 857-867.

${ }^{16}$ M. Ghulinyan, C. J. Oton, G. Bonetti, Z. Gaburro, and L. Pavesi, J. Appl. Phys. 93, 9724 (2003).

${ }^{17}$ I. M. White and X. Fan, Opt. Express 16, 1020 (2008).

${ }^{18}$ G. Lérondel, R. Romestain, and S. Barret, J. Appl. Phys. 81, 6171 (1997).

${ }^{19}$ P. J. Reece, G. Lérondel, W. H. Zheng, and M. Gal, Appl. Phys. Lett. 81, 4895 (2002).
${ }^{20}$ D. W. Thompson, P. G. Snyder, L. Castro, L. Yan, P. Kaipa, and J. A. Woollam, J. Appl. Phys. 97, 113511 (2005).

${ }^{21}$ G. Sulka and K. Hnida, Nanotechnology 23, 75303 (2012).

${ }^{22}$ T. Kumeria, M. M. Rahman, A. Santos, J. Ferré-Borrull, L. F. Marsal, and D. Losic, Anal. Chem. 86, 1837 (2014).

${ }^{23}$ H. Masuda and K. Fukuda, Science (80-.) 268, 1466 (1995).

${ }^{24}$ R. Ashtiani and P. Karami, Model. Numer. Simul. Mater. Sci. 5, 52924 (2015).

${ }^{25}$ H. Looyenga, Physica 31, 401 (1965).

${ }^{26}$ W. Theiss, Surf. Sci. Rep. 29, 91 (1997).

${ }^{27}$ M. J. Sailor, Porous Silicon in Practice: Preparation, Characterization and Applications (Wiley-VCH Verlag, 2012).

${ }^{28}$ E. Osorio, R. Urteaga, H. Juárez, and R. R. Koropecki, Sens. Actuators, B 213, 164 (2015).

${ }^{29} \mathrm{~F}$. Pedrotti, Introduction to Optics (Prentince-Hall, Inc., 1993).

${ }^{30}$ M. Lee and P. M. Fauchet, Opt. Express 15, 4530 (2007).

${ }^{31}$ L. C. Lasave, R. Urteaga, R. R. Koropecki, V. D. Gonzalez, and R. D. Arce, Colloids Surf., B 111, 354-359 (2013).

${ }^{32}$ J. Charrier and M. Dribek, J. Appl. Phys. 107, 044905 (2010).

${ }^{33}$ L. N. Acquaroli, R. Urteaga, and R. R. Koropecki, Sens. Actuators, B 149, 189 (2010).

${ }^{34}$ F. Vollmer and L. Yang, Nanophotonics 1, 267-291 (2012).

${ }^{35}$ E. W. Max Born, Principles of Optics (Cambridge University Press, 1980).

${ }^{36}$ C. K. Madsen and J. Zhao, Optical Filter Design and Analysis (John Wiley and Sons, Inc., 1999).

${ }^{37}$ B. E. Saleh and M. C. Teich, Fundamentals of Photonics (Wiley, 1991).

${ }^{38}$ J. D. Joannopoulos, S. G. Johnson, J. N. Winn, and R. D. Meade, Photonic Crystals, Molding the Flow of Light (Princeton University Press, 2008).

${ }^{39}$ T. Masuda, H. Asoh, S. Haraguchi, and S. Ono, Mater. (Basel). 8, 1350 (2015).

${ }^{40}$ L. N. Acquaroli, R. Urteaga, C. L. A. Berli, and R. R. Koropecki, Langmuir 27, 2067-2072 (2011). 